\title{
A INFLUÊNCIA DOUSO DO “CLAMP” OU BRAÇADEIRA NO ACÚMULO DE COÁGULOS EM DRENOS PLEURAIS TUBULARES
}

\author{
THE INFLUENCE OF CLAMP USAGE ON CLOT DEPOSIT FORMATION INSIDE \\ THORACIC DRAINS
}

\author{
Alexandre Garcia de Lima'; Elen Renate Figueira Rocha²; José Cláudio Teixeira Seabra ${ }^{3}$; \\ Ricardo Kalaf Mussi ${ }^{4}$; José Geraldo dos Santos ${ }^{5}$; Ivan Felizardo Contrera Toro ${ }^{6}$
}

\begin{abstract}
RESUMO: Objetivo: Conduziu-se este estudo prospectivo a fim de avaliar-se a influência do uso da braçadeira sobre o acúmulo de coágulos dentro dos drenos pleurais. Método: Os drenos pleurais foram pesados logo após sua retirada, lavados e secados e pesados novamente. A diferença entre a primeira e a segunda pesagem foi admitida como a quantidade de coágulos acumulada. Resultados: Houve maior acúmulo de coágulo nos drenos temporariamente obstruídos por braçadeira em relação àqueles não obstruídos. Conclusão: Notou-se, neste estudo, maior acúmulo de coágulo dentro de drenos pleurais obstruídos, mesmo que intermitentemente, o que pode levar ao mau funcionamento de todo o sistema de drenagem. A discussão sobre o correto uso dos drenos pleurais deve ser constante e fazer parte de programas de educação continuada para médicos e enfermagem, a fim de que este sistema, amplamente utilizado e altamente eficiente, seja otimizado (Rev. Col. Bras. Cir. 2008; 35(2): 079-082).
\end{abstract}

Descritores: Pleura; Cavidade pleural; Toracostomia; Tubos torácicos/efeitos adversos.

\section{INTRODUÇÃO}

A drenagem pleural tubular fechada é o meio mais usado para reexpansão pulmonar, evacuação da cavidade pleural e restabelecimento da pressão negativa intrapleural, seja em pós-operatórios eletivos ou em urgências pleurais, traumáticas ou não ${ }^{1-3}$.

O sistema de fluxo unidirecional mais comumente usado nas drenagens pleurais é o selo de água, ou drenagem subaquática ${ }^{3,4}$. Apesar de ser um dispositivo com mais de 100 anos $^{5}$ e amplamente utilizado, o seu correto mecanismo de funcionamento e seu manejo ainda são desconhecidos em muitos serviços, mesmo naqueles com especialistas da área da Medicina Torácica ${ }^{6,7}$.

O uso indiscriminado da braçadeira talvez seja o mais sério destes erros de manejo, pois a oclusão do dreno pleural através da braçadeira é um procedimento proscrito e que deve ser desencorajado por todos médicos, para toda equipe ${ }^{2,3,7-13}$ No entanto, por vício de uso ou por falta de informação, muitos serviços continuam a usar rotineiramente este dispositivo, para mobilização ou transporte, deambulação ou saída do leito, a despeito das orientações médicas e educação continuada.

$\mathrm{O}$ uso da braçadeira pode ter conseqüências agudas catastróficas, como pneumotórax hipertensivo, parada cardí- aca e hemotórax retido. No entanto, além dessas complicações, muito pouco se sabe acerca da influência da interrupção intermitente dos drenos tubulares pleurais sobre o funcionamento em médio prazo.

Sabe-se que o fluxo de líquidos através de uma estrutura tubular obedece à lei de Poiseuille ${ }^{6}$, sendo determinado, desta forma, pelo diâmetro e pela extensão do sistema. Por outro lado, a interrupção do fluxo, mesmo que momentaneamente, aumentaria a possibilidade de formação de coágulos dentro do dreno, o que poderia por sua vez, aumentar a possibilidade de oclusão e de mau funcionamento.

Para avaliar esta teoria desenvolveu-se este trabalho, realizado conjuntamente entre o Hospital das Clínicas da Unicamp e o Hospital Estadual Sumaré da Unicamp.

\section{MÉTODO}

Foram incluídos prospectivamente neste estudo todos os doentes submetidos à drenagem pleural tubular fechada em selo de água, atendidos na Disciplina de Cirurgia Torácica do Hospital das Clínicas da Unicamp e no Serviço de Cirurgia Torácica do Hospital Estadual Sumaré da Unicamp, no período de julho a dezembro de 2006.

\footnotetext{
1. Médico Cirurgião Torácico; Mestre em Cirurgia; Doutorando em Cirurgia Torácica e Cardiovascular; Médico Responsável pelo Serviço de Cirurgia Torácica e Endoscopia Respiratória do Hospital Estadual Sumaré.

2. Médica Residente do quarto ano de Operações Torácica do Hospital das Clínicas da Unicamp.

3. Médico Cirurgião Torácico; Médico Assistente da Disciplina de Operações Torácica do HC-UNICAMP.

4. Médico Cirurgião Torácico; Doutor em Cirurgia; Médico Assistente da Disciplina de Operações Torácica do HC-UNICAMP.

5. Médico Cirurgião Torácico; Professor Assistente da Disciplina de Operações Torácica do HC-UNICAMP.

6. Médico Cirurgião Torácico; Doutor em Cirurgia; Chefe da Disciplina de Operações Torácica do Hospital das Clínicas da Universidade Estadual de Campinas.
}

Recebido em 05/10/2007

Aceito para publicação em 05/12/2007

Conflito de interesses: nenhum

Fonte de financiamento: nenhuma

Trabalho realizado no Hospital das Clínicas da Universidade Estadual de Campinas - Unicamp e no Hospital Estadual Sumaré - Unicamp. 
Os doentes submetidos ao atendimento no Hospital das Clínicas tinham seus drenos inseridos/posicionados e as braçadeiras não retiradas, pois eram usadas pela equipe de enfermagem e do transporte, rotineiramente, para a oclusão temporária dos drenos quando os pacientes saiam do leito, trocavam de maca ou de leito, iam ao banheiro, deambulavam e também quando eram transportados às diversas unidades e setores do complexo hospitalar.

Já naqueles doentes atendidos no Hospital Estadual Sumaré, após a inserção do dreno, antes da conexão do dreno tubular ao sistema coletor/selo de água, a braçadeira era removida, não possibilitando assim o seu uso.

Todos os drenos eram de calibre 38 French, multifenestrados, com extensão de $120 \mathrm{~cm}$ e coletor de $2000 \mathrm{~mL}$.

Após a retirada dos drenos pleurais, todos foram submetidos à pesagem imediata. $\mathrm{O}$ peso do dreno com coágulos em seu interior era aferido com balança de precisão centesimal em gramas (denominado assim como Peso Úmido). O dreno então era limpo e seco e novamente pesado (denominado assim como Peso Seco). A diferença entre o Peso Úmido e o Peso Seco foi admitida como sendo a quantidade de coágulos acumulados dentro do dreno tubular pleural.

Foram excluídos deste estudo os drenos que foram submetidos à aspiração negativa, drenos dobrados e mal locados, bem como os utilizados para drenagem de pneumotórax simples, piotórax ou exsudatos pleurais e drenos utilizados para realização de pleurodese.

A análise estatística foi realizada com o programa estatístico Epi-Info versão 6,04d do Centro de Controle e Prevenção de Doenças, EUA, e da Organização Mundial de Saúde, Suíça. O teste utilizado foi o t de Student não pareado, com índice de significância de 0,05 .

Este estudo foi aprovado pelo Comitê de Ética em Pesquisa da Faculdade de Ciências Médicas da Universidade Estadual de Campinas através do Parecer 281/2006 e todos os doentes concordaram com o procedimento e com o estudo através de Termo de Consentimento Livre e Informado.

\section{RESULTADOS}

Foram inicialmente incluídos 65 drenos pleurais tubulares multifenestrados; destes, 12 foram excluídos por um dos motivos já citados. A amostra definitiva foi de 53 drenos pleurais, sendo 29 no grupo sem braçadeira e 24 no grupo com braçadeira. A idade dos doentes variou entre 17 e 76 anos (média 40,77 \pm 17,81 anos), sendo $17(32,1 \%)$ do gênero feminino. Não houve diferença quanto à idade $(\boldsymbol{p}=\mathbf{0 , 2 8})$ e quanto ao sexo $(\boldsymbol{p}=\mathbf{0 , 4 4})$ entre os grupos.

O tempo de drenagem variou entre 1 e 9 dias, com média de 3,26 $\pm 0,26$ dias. Não houve diferença entre o tempo de drenagem entre os grupos $(\boldsymbol{p}=\mathbf{0 , 7 6})$.

A diferença entre o peso úmido e o peso seco dos drenos foi de zero a 5,06 gramas, com média 1,20 $\pm 0,16$ gramas. Quando analisamos separadamente os grupos, observamos naquele com braçadeira uma variação de 0,13 a 5,06 g, média de $1,67 \pm 0,27$, e naqueles sem braçadeira uma variação de 0 a $3,38 \mathrm{~g}$, com média $0,81 \pm 0,17$ (Figura 1 ), sendo esta diferença foi significante $(\boldsymbol{p}=\mathbf{0 , 0 0 9})$ (Figura 2$)$.
Houve mais pacientes vítimas de traumatismos torácicos no grupo tratado sem braçadeira $(\boldsymbol{p}=\mathbf{0 , 0 0 0 8})$, mas número semelhante de drenagens em operações eletivas.

\section{DISCUSSÃO}

A drenagem pleural tubular subaquática ou em selo de água foi descrita, nos moldes como usada atualmente, em 1875 por Playfair ${ }^{5,10}$. Um sistema que se mantém da mesma forma por mais de um século e que, não obstante às frenéticas inovações tecnológicas na área médica, mantém-se o mesmo, é algo que impressiona e que advoga a favor de sua eficiência. No entanto seu correto manejo não é padronizado ${ }^{14-16}$, apesar da freqüente discussão na literatura médica especializada ${ }^{17}$.

Estes vícios de conduta podem ser explicados em serviços onde a rotina estabelecida não é discutida, e onde não se fazem cursos de reciclagem com toda a equipe, ficando, desta forma, condutas errôneas mantidas, a despeito da respeitabilidade da instituição.

O uso do "clamp" ou braçadeira no dreno pleural tubular em selo de água talvez seja o erro mais comum e mais amplamente difundido como "correto". No entanto a oclusão temporária através de braçadeira pode ter conseqüências imediatas catastróficas, como pneumotórax hipertensivo, balanço mediastinal, parada cardíaca e hemotórax retido.

O acúmulo de coágulo dentro de drenos pleurais acarreta preocupação entre cirurgiões cardiotorácicos ${ }^{18}$, e muitos

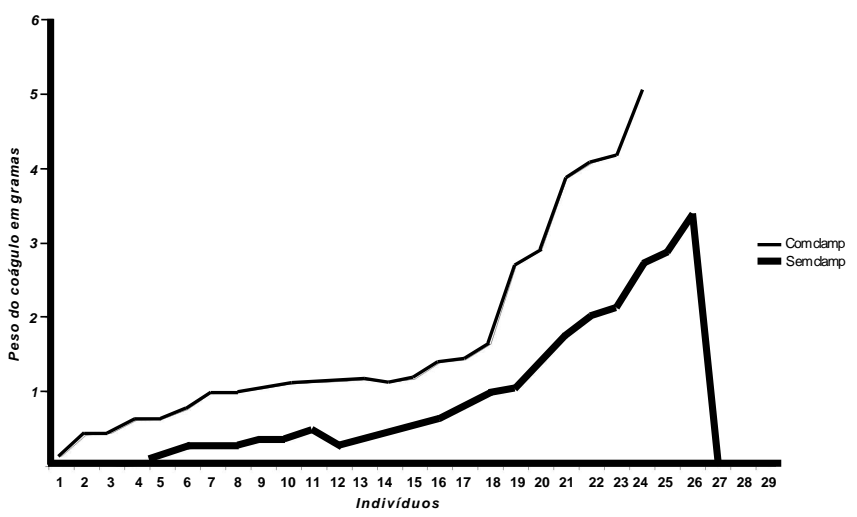

Figura 1 - Peso do coágulo acumulado dentro dos drenos pleurais com e sem o uso da braçadeira ou "clamp".

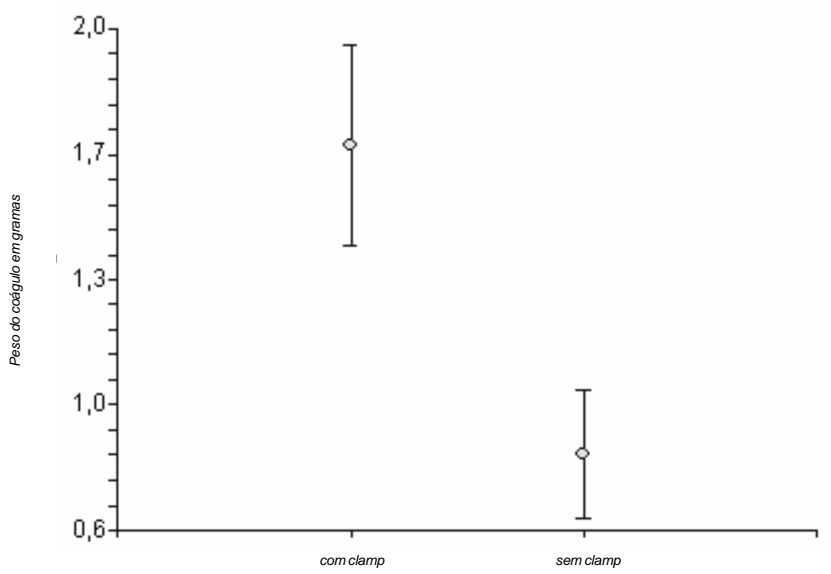

Figura 2 - Médias com desvio-padrão dos pesos dos coágulos, com e sem uso do "clamp". 
serviços têm rotinas de manuseio destes drenos a fim de prevenir sua oclusão.

O plasma e o sangue, evacuados através do dreno pleural, com sua alta concentração de proteínas, fibrina e fatores de coagulação, se submetidos ao fluxo com estase, e não contínuo, tendem a coagular com maior facilidade ${ }^{19}$. Ora, este fluxo interrompido, mesmo que momentaneamente, é a conseqüência do uso da braçadeira no dreno pleural.

Os drenos pleurais funcionam através dos seguintes mecanismos ${ }^{10}$ :

1 - Variação pressórica intrapleural durante o ciclo respiratório: sabe-se que a pressão intrapleural pode variar durante um ciclo respiratório normal entre $-2 \mathrm{~cm} \mathrm{H}_{2} \mathrm{O}-8 \mathrm{~cm} \mathrm{H}_{2} 0$, ao final da expiração e inspiração, respectivamente, podendo alcançar valores de $+70 \mathrm{~cm} \mathrm{H}_{2} 0 \mathrm{e}-54 \mathrm{~cm} \mathrm{H}_{2} 0^{10}$, em esforços de tosse ou em inspiração profunda, respectivamente. Se considerarmos o doente com drenagem pleural no leito, em repouso, estas pressões intrapleurais vão tender sempre para valores negativos, e o aumento da pressão intrapleural, que levaria ao aumento do efluxo através do dreno, não é conseguido. Este efluxo é aumentado justamente quando o doente esforça-se no leito, para levantar-se ou fazer suas atividades, deambular pelos corredores ou tomar banho, ou simplesmente conversar (o que aumenta a pressão intrapleural para $+10 \mathrm{~cm} \mathrm{H}_{2}$ ). Se o dreno, no entanto, estiver ocluído nestes momentos, perde-se a oportunidade de drenagem efetiva;

2 - Sistema de vasos comunicantes: o fluxo de líquidos entre um sistema de vasos comunicantes dá-se no sentido do recipiente mais elevado em direção ao inferior, razão pela qual o frasco coletor/selo de água deve ficar em desnível em relação ao doente. Suponhamos que o doente seja transferido ao setor de radiologia, e que precisamos acomodar o frasco entre as pernas do doente. Neste momento não é necessária a oclusão do dreno, pois, na pior das hipóteses, o frasco encontra-se no mesmo nível do hemitórax do paciente, havendo portanto um equilíbrio entre as pressões. Levando-se em conta ainda a variação de pressão no sistema, durante o ciclo respiratório, sabemos que a máxima pressão negativa é de $54 \mathrm{~cm}$ de $\mathrm{H}_{2} 0$, durante uma inspiração profunda; ora, o sistema do dreno, com tubo de extensão, mede cerca de $200 \mathrm{~cm}$, o que gera uma margem de segurança de cerca de $150 \mathrm{~cm}$. Desta forma, mesmo que o doente inspire profundamente durante todo o transporte, a coluna irá subir no máximo $54 \mathrm{~cm}$ de $\mathrm{H}_{2} 0$. Portanto, se durante o transporte ou mobilização dos doentes, tomar-se o devido cuidado para que o frasco não seja elevado além da altura do hemitórax, o dreno não precisa, e não deve, ser obstruído;

3 - Capilaridade: devido a multifenestração dos drenos tubulares, o fluxo foi favorecido, mas talvez este seja o meca- nismo menos importante para o fluxo de líquidos da cavidade pleural para o frasco.

Paralelamente, durante a troca do selo de água, perde-se o sistema de fluxo unidirecional, que é o selo de água, e o sistema fica aberto, com comunicação direta do meio externo com a cavidade pleural. Mas mesmo neste momento, a oclusão pode ser evitada, ou por troca da conexão por outro selo de água previamente confeccionado, ou por oclusão do sistema não com a braçadeira, mas com a mão, o que impede que o sistema continue fechado após a conexão ao novo selo de água. Um erro freqüente no manejo do dreno é obstruí-lo temporariamente, por quaisquer motivos, e não desobstruí-lo logo após, ficando o sistema todo fechado por longos períodos.

Nos EUA existem duas marcas de drenos pleurais que vêm com braçadeiras, e que não são recomendadas pela maioria dos serviços justamente por este motivo ${ }^{10}$.

Neste estudo, apesar das amostras serem provenientes de dois hospitais distintos, consideraram-se os dados comparáveis, visto que os drenos utilizados foram semelhantes, pois a equipe de Cirurgia Torácica é a mesma e as equipes de enfermagem e de fisioterapia passam pelo mesmo treinamento, proveniente do Hospital das Clínicas da Unicamp. Desta forma, demonstrou-se claramente a desvantagem do uso do sistema de braçadeira para oclusão temporária dos drenos pleurais, com maior acúmulo de coágulos sanguíneos no sistema quando comparados àqueles não ocluídos. Esse acúmulo de coágulos gera mau funcionamento do sistema, drenagem ineficiente e conseqüentemente complicações pleurais em conseqüência ao mau uso dos drenos.

Pretende-se prosseguir nesta linha de pesquisa com grupos mais homogêneos, somente com drenagem por traumatismo ou somente em operaçõess eletivas, bem como manter estes pacientes em seguimento clínico para estudarem-se as complicações pleurais precoces e tardias.

Conclui-se que o uso da braçadeira como rotina no dia-a-dia do manejo do dreno pleural subaquático demonstrou-se mais uma vez danoso e desnecessário, devendo ser, por mais este motivo, desencorajado e proscrita.

\section{Agradecimentos}

Agradecemos à colaboração dos laboratórios de patologia clínica do Hospital Estadual Sumaré e do Hospital das Clínicas da Unicamp por ceder seus equipamentos de precisão e seu espaço físico. Congratulamo-nos com a equipe de enfermagem do Hospital Estadual Sumaré pela predisposição à discussão e à educação continuada.

\begin{abstract}
Background: A prospective study was done to evaluate the influence of clamp usage on clot formation inside thoracic drains. Methods: Each drain was weighed soon after removal; they were then washed, dried and weighed again. The difference between the first and second weights was taken as the amount of clot deposit formed. Results: We found more clots accumulated inside the drains that were temporarily obstructed by the clamp. Conclusion: In this study, there were more clots formation inside thoracic drains clamped, even if they were occluded intermittently. This can lead thoracic drains to function improperly. The discussion about the correct usage of thoracic drains must be a subject for educational programs for physicians and nurses, to aim for the safest use of this widely used and highly efficient system.
\end{abstract}

Key words: Pleura; Pleural cavity; Thoracostomy; Chest tubes/adverse effects. 


\section{REFERÊNCIAS}

1. Lima AG, Toro IFC, Tincani AJ, Barreto G. A drenagem pleural pré-hospitalar: apresentação de mecanismo de válvula unidirecional. Rev Col Bras Cir. 2006; 33(2):101-6.

2. Barton ED, Epperson M, Hoyt DB, Fortlage D, Rosen P. Prehospital needle aspiration and tube thoracostomy in trauma victims: a six-year experience with aeromedical crews. J Emerg Med. 1995; 13(2):155-63.

3. Hyde J, Sykes T, Graham T. Reducing morbidity from chest drains. BMJ. 1997; 314(7085):914-5.

4. McMahon-Parkes K. Management of pleural drains. Nurs Times. 1997; 93(52):48-9.

5. Wagner RB, Slivko B. Highlights of the history of nonpenetranting chest trauma. Surg Clin North Am. 1989; 69(1):1-14.

6. Baumann MH. What size chest tube? What drainage system is ideal? And other chest tube management questions. Curr Opin Pulm Med. 2003; 9(4):276-81.

7. Harris DR, Graham TR. Management of intercostal drains. Br J Hosp Med. 1991; 45(6):383-6.

8. Graham AN, Cosgrove AP, Gibbons JRP, McGuigan JA. Randomised clinical trial of chest drainage systems. Thorax. 1992; 47(6):461-2.

9. Symbas PN. Chest drainage valve. Emerg Med Serv. 1978; 7(3):41-6.

10. Munnell ER. Thoracic drainage. Ann Thorac Surg. 1997; 63(5):1497-502.

11. Coughlin AM, Parchinsky C. Go with the flow of chest tube therapy. Nursing. 2006; 36(3):36-41.

12. Shuster PM. Chest tubes: to clamp or not to clamp. Nurs Educ. 1998; 23(3):9-13.
13. Tooley C. The management and care of chest drains. Nurs Times Plus. 2002; 98(26):49-50.

14. Bar-El Y, Ross A, Kablawi A, Egenburg S. Potentially dangerous negative intrapleural pressures generated by ordinary pleural drainage systems. Chest. 2001; 119(2):511-4.

15. Smith RN, Fallentine J, Kessel S. Underwater chest drainage: bringing the facts to the surface. Nursing. 1995; 25(2):60-3.

16. Younes RN, Gross JL, Aguiar S, Haddad FJ, Deheinzelin D. When to remove a chest tube? A randomised study with subsequent prospective consecutive validation. J Am Coll Surg. 2002; 195(5):658-62.

17. Gambazzi F, Schirren J. Thoraxdrainagen. Was its "evidence based"? Chirurg. 2003; 74(2):99-107.

18. Wallen M, Morrison A, Gillies D, O'Riordan E, Brigde C, Stoddart F. Mediastinal chest drain clearance for cardiac surgery. The Cochrane Library, Issue 1, 2006.

19. Tattersall DJ, Traill ZC, Gleeson FV. Chest drains: does size matter? Clin Radiol. 2000; 55(6):415-21.

Como citar este artigo:

Lima AG, Rocha ER, Seabra JC, Mussi RK, Santos JG, Contrera Toro IF. A influência do uso do "clamp" ou braçadeira no acúmulo de coágulos em drenos pleurais tubulares. Rev Col Bras Cir. [periódico na Internet] 2008; 35(2). Disponível em URL: http://www.scielo.br/ rcbc

Endereço para correspondência:

Alexandre Garcia de Lima

Rua João Simões da Fonseca, $\mathrm{n}^{\circ} 70$

Cond. Residencial Barão do Café 4

13085-050 - Campinas - SP

Tel.: (19) 3289-3573 - 9605-5448

E-mail:dralexandregarcia@terra.com.br 